\title{
Papers
}

\section{Which contacts of patients with meningococcal disease carry the pathogenic strain of Neisseria meningitidis? A population based study}

\author{
Bjørn-Erik Kristiansen, Yngvar Tveten, Andrew Jenkins
}

\begin{abstract}
Objectives: To determine the prevalence of the pathogenic strain of Neisseria meningitidis in contacts of patients with meningococcal disease, and to determine which contact groups are likely to be carriers and warrant chemoprophylaxis.

Design: Population based study. Setting: Norwegian county of Telemark.

Subjects: 1535 primary contacts of 48 patients with meningococcal disease, and 78 secondary contacts.

Interventions: Carriers of the pathogenic strain were treated with rifampicin. All household members and kissing contacts under 15 years of age were treated with oral penicillin. Contacts were taught to recognise the symptoms of meningococcal disease.

Results: In 27 of 48 cases investigated, contacts carrying the pathogenic strain of $N$ meningitidis were found. A total of 42 such contacts were identified. Contacts were stratified into three classes according to the assumed closeness of contact with patients. In class 1 (household members and kissing contacts) the prevalence of the pathogenic strain was $12.4 \%$ (95\% confidence interval $5.5 \%$ to $19.3 \%$ ). In classes 2 and 3 the prevalence was $1.9 \%(0.9 \%$ to $3.4 \%)$ and $1.6 \%$ $(0.14 \%$ to $3.1 \%)$.

Conclusions: There is a high rate of carriage of the pathogenic strain of $N$ meningitidis in patients' household members and kissing contacts, and this supports the practice of giving chemoprophylaxis to these contacts. The prevalence of carriage among other contacts is 2-3 times that found in the general population $(0.7 \%)$; the benefits of chemoprophylaxis to these contacts may be marginal.
\end{abstract}

\section{Introduction}

Contacts of patients with meningococcal disease have an increased risk of contracting the disease (relative risk for household members between 1000 and 4000). ${ }^{1-3}$ When meningococcal disease occurs, often carriers of the pathogenic strain of Neisseria meningitidis can be found in the patient's contacts. ${ }^{4-6}$ These carriers may develop the disease or the bacterium may spread from person to person eventually causing disease in someone without apparent link with the first patient. The frequency of secondary or associated cases has been reported as $0.5 \%{ }^{7}$ However, estimates may be higher if the time interval is extended ${ }^{7}$ or if epidemiological studies using sensitive identification techniques for bacterial strains are applied. ${ }^{589}$ One study found 22 $(9.5 \%)$ associated cases among 220 cases of meningococcal disease in Norway during 1994-96 using such methods. ${ }^{10}$

To prevent the spread of meningococcal infection, the World Health Organisation and the health authorities of most countries recommend that close contacts should receive chemoprophylaxis to eradicate the pathogenic strain. ${ }^{11-14}$ However, it may be difficult to define who is a close contact, and still more difficult to define who should be excluded from this definition. Therefore chemoprophylaxis is often given to more contacts than is needed. ${ }^{15}$ In Norway, before 1970 , liberal sulphonamide chemoprophylaxis was practised. However, the emergence of a virulent clone of $\mathrm{N} \mathrm{men-}$ ingitidis that was resistant to sulphonamide ${ }^{16}$ led the Norwegian authorities to abandon chemoprophylaxis for fear of further resistance problems. Instead, household members under 15 years of age are assumed to have meningococcal disease and are treated with penicillin orally for 1 week. ${ }^{17}$

Since November 1987 we have run the Telemark meningococcal project in which rifampicin prophylaxis is targeted to carriers of the pathogenic strains of $N$ meningitidis identified by DNA fingerprinting of nasopharyngeal meningococci. Secondary cases have not been observed. ${ }^{5}$ We used data from this project to address the questions: "Who is most likely to carry the pathogenic strain of $N$ meningitidis after a case of meningococcal disease?" and "To whom should chemoprophylaxis be restricted?"

\section{Subjects and methods}

The Telemark project

The detailed organisation of this project has been described previously. ${ }^{5}$ After isolation of meningococci from a patient specimen the local health officer is alerted, who then collects throat samples from members of the patient's household before initiating penicillin treatment to those under 15 years of age. ${ }^{17}$ Parents accompanying the patient to hospital are often sampled by the hospital staff.

\author{
A/S Telelab, \\ Telemark \\ Biomedical Centre, \\ PO Box 1868 \\ Gulset, N-3705 \\ Skien, Norway \\ Yngvar Tveten, \\ consultant \\ Andrew Jenkins, \\ researcher \\ Department of \\ Medical \\ Microbiology, \\ University of \\ Tromsø, 9037 \\ Tromsø, Norway \\ Bjørn-Erik \\ Kristiansen, \\ professor \\ Correspondence to: \\ Dr Kristiansen \\ bjorneri@online.no
}

BMJ 1998;317:621-5 


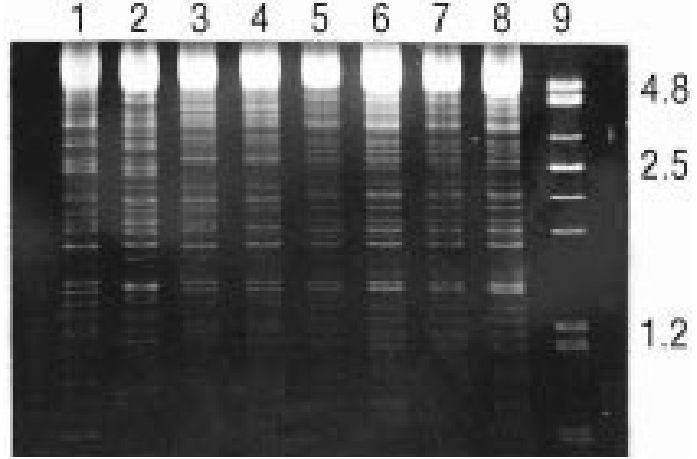

Chromosomal DNA fingerprints of meningococcal isolates from patient (lane 3) and contacts (lanes 1, 2, and 4-8). The size marker (lane 9) is a Pstl digest of lambda DNA (sizes in kb). Isolate from patient's kissing contact (lane 4) identical with that of patient

Based on information from people who know the patient a list of close contacts is drawn up; throat samples are collected from these contacts on the same or next day. Simultaneously, the local community is informed about the disease in open meetings. When a pathogenic strain is found, rifampicin prophylaxis ( $600 \mathrm{mg}$ twice daily for two days; children $<12$ years of age $10 \mathrm{mg} / \mathrm{kg}$ ) is given and throat samples collected from the contact's household and kissing contacts (secondary contacts).

Table 1 Details of 48 patients who presented with meningococcal disease in Telemark, Norway from 1 November 1987 to 1 December 1996

\begin{tabular}{|c|c|c|c|c|c|c|}
\hline \multirow[b]{2}{*}{ Age (years) } & \multicolumn{4}{|c|}{ Serogroup } & \multirow{2}{*}{$\begin{array}{l}\text { No of } \\
\text { patients }\end{array}$} & \multirow{2}{*}{$\begin{array}{l}\text { No of patients with contacts } \\
\text { carrying pathogenic strain }\end{array}$} \\
\hline & B & C & $Y$ & W135 & & \\
\hline $0-4$ & 16 & 8 & - & - & 24 & 14 \\
\hline $5-12$ & 4 & 1 & 1 & - & 6 & 5 \\
\hline $13-18$ & 3 & 4 & 2 & - & 9 & 5 \\
\hline $19-60$ & 5 & - & - & - & 5 & 1 \\
\hline$>60$ & 2 & 1 & - & 1 & 4 & 2 \\
\hline Total & 30 & 14 & 3 & 1 & 48 & 27 \\
\hline
\end{tabular}

Table 2 Bacteriological findings in class 1 contacts (household members and kissing contacts)

\begin{tabular}{lcccc} 
Group & Contact & Total No & No (\%) of carriers & $\begin{array}{c}\text { No (\%) of carriers with } \\
\text { pathogenic strain }\end{array}$ \\
\hline 1 & Father & 37 & $9(24)$ & $5(14)$ \\
\hline 2 & Mother & 37 & $9(24)$ & $6(16)$ \\
\hline 3 & Sister & 21 & $2(10)$ & $1(5)$ \\
\hline 4 & Brother & 29 & $6(21)$ & $4(14)$ \\
\hline 5 & Kissing & 3 & $1(33)$ & $1(33)$ \\
\hline All groups & Others & 18 & $9(50)$ & $1(6)$ \\
\hline
\end{tabular}

Table 3 Bacteriological findings in class 2 contacts

\begin{tabular}{llrcc} 
Group & \multicolumn{1}{c}{ Contacts } & Total No & $\begin{array}{c}\text { No (\%) of } \\
\text { carriers }\end{array}$ & $\begin{array}{c}\text { No (\%) of carriers with } \\
\text { pathogenic strain }\end{array}$ \\
\hline 7 & Grandparents & 41 & $6(15)$ & $2(5)$ \\
\hline 8 & Others* & 37 & $5(14)$ & 0 \\
\hline 9 & Playmates, close friends & 249 & $50(20)$ & $5(2)$ \\
\hline 10 & Nursery employees & 60 & $5(9)$ & $1(2)$ \\
\hline 11 & Childminders, babysitters, home helps & 5 & $2(40)$ & $1(20)$ \\
\hline 12 & Other family & 184 & $37(20)$ & $2(1)$ \\
\hline Total & & 576 & $105(18.2)$ & $11(1.9)$ \\
\hline
\end{tabular}

*Playmates' family and childminders' children.

†Parents, children, and siblings outside the patient's household, and cousins, fathers in law, mothers in law, sons in law, and daughters in law.
For each contact a standard questionnaire is completed for personal data, type of contact with the patient, and symptoms of respiratory disease.

Collection of throat samples-Both tonsils and the posterior pharyngeal surface were sampled with a cotton swab, plated immediately on GC agar base (Mast Diagnostics, Merseyside, UK) supplemented with haematin, 1\% IsoVitalex (BBL, Cockeysville, MD,USA), vancomycin $(3 \mathrm{mg} / \mathrm{l})$, and colistin $(7.5 \mathrm{mg} / \mathrm{l})$, and incubated at $37^{\circ} \mathrm{C}$ in $10 \%$ carbon dioxide within two hours of sampling. Wherever possible, sampling was done by two of the authors (BK and YT).

Identification of the pathogenic strain-Contacts carrying a meningococcus with a chromosomal DNA fingerprint identical to that of the patient isolate were identified as previously described ${ }^{18}$ and were defined as carrying the pathogenic strain (fig).

\section{Statistical methods}

Confidence intervals for the prevalence of the pathogenic strain were calculated as follows:

The parameter of interest is the probability, p, of carrying the pathogenic strain.

$\mathrm{m}$ is the number of patients diagnosed. Assume that patient $i$ has $n_{i j}$ contacts in class $j$, and that each of them has probability $\mathrm{p}_{\mathrm{ij}}$ of carrying a pathogenic strain. The number, $\mathrm{X}_{\mathrm{ij}}$, of the $\mathrm{n}_{\mathrm{ij}}$ contacts carrying the pathogenic strain is a binomial $\left(n_{i j} p_{i j} ; i=1,2, \ldots m ; j=I\right.$, II, III).

Then $p_{j}$, the probability of carriage for each of the three contact classes, is estimated by the weighted average of the $p_{i j}$ 's:

$$
\hat{p}_{j}=\sum_{i=1}^{m} X_{i j} / \sum_{t=1}^{m} n_{i j} ; j=I, I I, I I I
$$

which has a statistical mean $\mathrm{p}_{\mathrm{j}}$ and a statistical variance estimated by

$$
\frac{1}{N_{j}^{2}} \sum_{t=1}^{m} n_{i j} \hat{p}_{i j}\left(1-\hat{p}_{i j}\right)
$$

Since the $n_{i j}$ 's and $X_{i j}$ 's are small, the $\hat{p}_{i j}$ 's are estimated by

$$
\hat{p}_{i j}=\frac{X_{i j}+1}{n_{i j}+2} ; i=1 \ldots m, j=I, I I, I I I
$$

Thus an approximate $95 \%$ confidence interval for $\mathrm{p}_{\mathrm{j}}$ is given by

$$
C I_{0.95}=\hat{p}_{j} \pm 1.96 \frac{1}{N_{j}}\left[\sum_{t=1}^{m} n_{i j} \hat{p}_{i j}\left(1-\hat{p}_{i j}\right]_{1 / 2}\right.
$$

\section{Results}

Disease characteristics-From 1 November 1987 to 1 December 1996 there were 48 cases (cases 3-50) of meningococcal disease in the county of Telemark, Norway, verified bacteriologically (table 1). Thirty isolates were serogroup $\mathrm{B}, 14$ were serogroup $\mathrm{C}$, three were serogroup Y, and one was serogroup W135. Twenty four of the patients were under 4 years of age. The remaining cases were distributed in the age groups 5-12 years (six cases), 13-18 years (nine), 19-60 years (five), and $>60$ years (four).

Classification of contacts into groups and classes-We collected throat specimens from 1535 close contacts (primary contacts) of the patients, and from 78 secondary contacts who were household members or kissing 
contacts of primary contacts found to carry the pathogenic strain. All contacts approached consented to sampling. The primary contacts were divided into 16 contact groups and further organised into three classes according to the degree of contact with the patient (tables 2 to 4). Class 1 consists of household members and kissing contacts, the groups of contacts assumed to have the closest contact with the patient. Class 2 contacts are considered to have closer contact with the patient than class 3 contacts. Secondary contacts were placed in a separate group, group 18 (table 5).

Carrier rate-Among 1535 primary contacts, 234 meningococcal carriers were found. Of these, 42 carried the pathogenic strain. Thirty six of the 145 class 1 contacts carried meningococci. The pathogenic strain was found in 18 (12.4\%: 95\% confidence interval $5.5 \%$ to $19.3 \%)$ of these contacts: $6 / 37$ (16.2\%) mothers, $5 / 37$ (13.5\%) fathers, 4/29 (13.8\%) brothers, $1 / 21(4.8 \%)$ sisters, $1 / 18(5.5 \%)$ other household members, and 1/3 (33.3\%) kissing contacts. Of 576 class 2 contacts, $105(18.2 \%)$ carried meningococci The pathogenic strain was found in $11(1.9 \%: 0.9 \%$ to $3.4 \%)$. Of 814 class 3 contacts, $93(11.4 \%)$ carried meningococci. The pathogenic strain was found in 13 (1.6\%: $0.14 \%$ to $3.1 \%$ ) of these contacts. Of 78 secondary contacts, $20(25.6 \%)$ carried meningococci. The pathogenic strain was found in four $(5.1 \%)$ of these contacts.

The pathogenic strain was found in the primary contacts of 27 of the 48 patients, the number of carriers varying between 1 and 6 (table 5). The pathogenic strain was found more often in contacts in the 5-12 years age group than in the other age groups (table 1).

\section{Discussion}

In most countries the use of chemoprophylaxis is recommended to prevent secondary disease in close contacts of patients with meningococcal disease. ${ }^{11-14}$ In a few other countries, including Norway, chemoprophylaxis is not recommended, but household members under 15 years of age are treated with penicillin orally. ${ }^{17}$ Neither approach has been evaluated in controlled studies.

When a case of meningococcal disease occurs, many people may fulfill accepted criteria for receiving chemoprophylaxis, ${ }^{11-14}$ and chemoprophylaxis may be prescribed in excess of what is needed. ${ }^{14}$ High consumption of chemoprophylactic agents may select bacterial resistance, which in meningococci may be associated with virulence. ${ }^{19}$ Chemoprophylactic agents may also kill non-virulent meningococci and other bacteria that stimulate an immune response against the meningococci. ${ }^{20}$ Chemoprophylaxis should therefore be restricted to those who are likely to carry the pathogenic strain.

Our study shows that only 42 (2.7\%) of 1535 close contacts carried the pathogenic strain of $N$ meningitidis. General use of chemoprophylaxis in all these contacts therefore seems excessive. Sensitive and rapid techniques for identification of the pathogenic strain ${ }^{8}$ allow targeting of chemoprophylaxis to carriers, but have not yet been widely applied as most laboratories lack the technology and resources to perform these tests. In most cases, therefore, the decision of whether
Table 4 Bacteriological findings in class 3 contacts

\begin{tabular}{llccc} 
Group & \multicolumn{1}{c}{ Contacts } & Total No & No (\%) of carriers & $\begin{array}{c}\text { No (\%) of carriers with } \\
\text { pathogenic strain }\end{array}$ \\
\hline 13 & Classmates & 286 & $51(18)$ & $4(1)$ \\
\hline 14 & Children at same nursery & 220 & $9(4)$ & $2(1)$ \\
\hline 15 & Teachers & 44 & $2(5)$ & 0 \\
\hline 16 & Others* $^{*}$ & 264 & $31(12)$ & $7(3)$ \\
\hline All groups & & 814 & $93(11.4)$ & $13(1.6)$ \\
\hline
\end{tabular}

*Neighbours, pupils at same school but not in patient's class, nurses, and doctors. Contacts sampled only when they had been in close contact with patient during past two weeks before patient became ill.

Table 5 Contacts carrying pathogenic strain. Age in years unless stated otherwise

contacts carrying pathogenic strain

\begin{tabular}{lccccc} 
& Sex (age) of & & Contact \\
\cline { 3 - 5 } Outbreak No & patient & Serogroup & Relation to patient (sex) & Age & \begin{tabular}{c} 
group \\
\hline
\end{tabular}
\end{tabular}

$\begin{array}{lccccc}03 & \text { Male (5) } & \text { B } & \text { Employee daycare centre (male) } & 26 & 10\end{array}$

\begin{tabular}{rllllr}
\hline & & Mother & 35 & 2 \\
\hline 04 & Female (11) & B & Father & 35 & 1
\end{tabular}

\begin{tabular}{llllcc}
\hline 04 & Female (11) & B & Father & 35 & 1 \\
\hline 05 & Male (2) & B & Brother & 6 months & 4 \\
\hline & & & Mother & 25 & 2
\end{tabular}

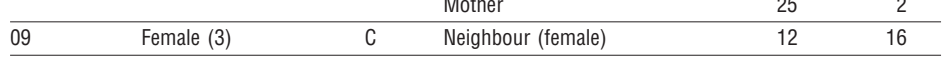

\begin{tabular}{llrr}
\hline & Playmate (male) & 4 & 16 \\
\hline
\end{tabular}

\begin{tabular}{|c|c|c|c|c|c|}
\hline & & & Playmate (male) & 4 & 16 \\
\hline & & & Neighbour (female) & 1 & 16 \\
\hline & & & Neighbour (female) & 2 & 16 \\
\hline & & & Neighbour (male) & 16 & 16 \\
\hline 12 & Male (5 months) & B & Father & 28 & 1 \\
\hline \multirow[t]{2}{*}{13} & Male (4) & $B$ & Brother & 16 & 4 \\
\hline & & & Mother & 37 & 2 \\
\hline \multirow[t]{2}{*}{14} & Male (11) & $\mathrm{Y}$ & Playmate (male) & 10 & 9 \\
\hline & & & Neighbour (male) & 7 & 16 \\
\hline \multirow[t]{2}{*}{17} & Female (18) & C & Friend (male) & 19 & 9 \\
\hline & & & Classmate (male) & 19 & 13 \\
\hline \multirow[t]{3}{*}{18} & Male (1) & C & Mother & 34 & 2 \\
\hline & & & Brother & 4 & 4 \\
\hline & & & Home help (female) & 23 & 11 \\
\hline 20 & Female (77) & W135 & Daughter & 45 & 12 \\
\hline 21 & Male (39) & $B$ & Kissing contact (male) & 44 & 6 \\
\hline 22 & Male (17) & B & Classmate (male) & 13 & 13 \\
\hline \multirow[t]{2}{*}{25} & $\begin{array}{l}\text { Female (8 } \\
\text { months) }\end{array}$ & B & Childminder (male) & 24 & 11 \\
\hline & & & Secondary contact (female) & 25 & 18 \\
\hline 26 & Male (1) & B & Grandmother & 41 & 7 \\
\hline 27 & Male (18) & $\mathrm{C}$ & Friend (male) & 18 & 9 \\
\hline 29 & Male (2) & B & Father & 48 & 1 \\
\hline 30 & Male (18) & $B$ & Best friend (male) & 20 & 9 \\
\hline 36 & Female (1) & $B$ & Grandmother & 56 & 7 \\
\hline \multirow[t]{4}{*}{38} & Male (16) & $\mathrm{Y}$ & Classmate 1 (male) & 16 & 13 \\
\hline & & & Classmate 2 (male) & 16 & 13 \\
\hline & & & Kissing contact of 1 (female) & 15 & 18 \\
\hline & & & Kissing contact of 2 (female) & 15 & 18 \\
\hline 39 & Male (5 months) & B & Father & 24 & 1 \\
\hline 41 & Male (1) & B & Father & 28 & 1 \\
\hline 43 & Male (4) & C & Mother & 36 & 2 \\
\hline \multirow[t]{2}{*}{44} & Male (3) & $B$ & Brother & 8 & 4 \\
\hline & & & Uncle & 35 & 12 \\
\hline 46 & Male (11) & B & Best friend (male) & 8 & 9 \\
\hline 47 & Male (15) & B & Kissing contact (female) & 14 & 5 \\
\hline \multirow[t]{2}{*}{48} & Male (4) & B & Mother & 35 & 2 \\
\hline & & & Sister & 6 & 3 \\
\hline \multirow[t]{2}{*}{50} & Female (6) & $B$ & Child 1 (male) & 6 & 14 \\
\hline & & & Brother of child 1 & 10 & 18 \\
\hline
\end{tabular}

to give chemoprophylaxis must be made on the basis of closeness of contact with the patient.

Our study shows that the risk of carriage of the pathogenic strain is highest (12.4\%, 95\% confidence interval $5.5 \%$ to $19.3 \%$ ) in household members and 
kissing contacts. Household members have a high relative risk of meningococcal disease $\left(1000-4000^{1-3}\right)$ and the use of chemoprophylaxis in this group therefore seems well justified. Contacts outside this group, most of whom qualified for chemoprophylaxis according to accepted criteria, ${ }^{11-14}$ had a considerably lower prevalence of carriage of the pathogenic strain (class 2, 1.9\%, $0.9 \%$ to $3.4 \%$; class $3,1.6 \%, 0.14 \%$ to $3.1 \%)$. This is higher than the $0.7 \%$ prevalence in the general population, during periods of low disease incidence, ${ }^{18}{ }^{21}$ but not dramatically so. Should these contacts receive chemoprophylaxis? Our results do not support this practice. However, the relative risk of meningococcal disease is over 1000 for household members, although our results indicate that the carriage rate $(12.4 \%)$ is only 18 times higher than that found in the general population $(0.7 \%)$ in other studies. The relative risk of meningococcal disease is therefore not a simple function of the prevalence of the pathogenic strain. Another way to view the problem would be to ask whether the prevalence of the pathogenic strain approaches that needed to initiate epidemic disease. It has been suggested that a high rate of carriage is a prerequisite for epidemic disease, ${ }^{22}$ but the threshold is not known, and in any case the prevalence will vary from case to case. We therefore feel that the choice of whether to give chemoprophylaxis to contacts outside the patient's household and kissing contacts should be made on an individual basis, taking into account: other cases in the vicinity or other reasons to suspect an outbreak in the community; a high incidence of influenza or other respiratory infection that may predispose contacts to meningococcal disease and mask the symptoms of early infection; and other predisposing factors. If, however, an isolated case occurs in an otherwise healthy community, we believe that a conservative approach to chemoprophylaxis is justified.

Our study illustrates the need for better understanding of the relation between carrier rate and risk of secondary disease. A controlled study comparing different chemoprophylaxis strategies would in our view be of considerable help. The following strategies should be considered: (a) chemoprophylaxis according to standard recommendations, (b) chemoprophylaxis given only to household members and kissing contacts, and (c) chemoprophylaxis given to household members, kissing contacts, and other close contacts who are found to carry the pathogenic strain. Cost benefit analysis and studies of the prevalence of the pathogenic strain would enhance the value of such a study.

It has been argued that throat swabbing underestimates the true rate of meningococcal carriage..$^{23}$ Low levels of bacteria in the sample, loss of viability under transport, and variable sampling techniques can all influence the measured carrier rate, but sampling and transport are probably the more important factors. ${ }^{24}$ We have addressed this problem by attempting to confine sampling to two well trained members of our staff, by plating samples directly after collection, and by sampling the throat, which has been reported to have $100 \%$ sensitivity relative to other sampling sites. ${ }^{25} \mathrm{~A}$ minority of the samples were collected by hospital and clinic staff and transported before plating. These are almost exclusively samples collected from members of the patient's household-that is, the group where we
- Contacts of patients with meningococcal disease have a $12.4 \%$ (95\% confidence interval $5.5 \%$ to $19.3 \%$ ) risk of carrying the pathogenic meningococcus if they are kissing contacts or household members

- The risk of carriage of the pathogenic strain for two groups of contacts less close than household members or kissing contacts is $1.9 \%$ $(0.9 \%$ to $3.4 \%)$ and $1.6 \%(0.14 \%$ to $3.1 \%)$

found the highest rates of carriage. Serious underestimation of carriage might be expected to lead to secondary cases among the contacts we sampled. None of the 1535 primary and 78 secondary contacts contracted meningococcal disease. We therefore do not think that sampling problems seriously affect this study.

Contributors: B-EK had the original idea for the present study, collected samples, and wrote the manuscript. Randi Kersten (A/S Telelab, Skien, Norway) analysed the data and wrote an initial manuscript as part of her magisterial dissertation in microbiology, Anne Gry Allum (A/S Telelab) assisted in sample collection and performed DNA fingerprinting, YT collected samples and helped with data analysis. AJ took an active part in the analysis and discussion of data, and in writing the manuscript. Statistical analysis was conducted by Tone Grande (Mericon A/S, Skien, Norway). A theoretical analysis of the problem of estimating confidence intervals in this data set was provided by Professor Nils Lid Hjort, University of Oslo. The Telemark project was started by Arne-Birger Knapskog, country health officer, Skien, Norway. B-EK and AJ will act as guarantors for the paper.

Funding: This project is a part of A/S Telelab's internally funded research programme.

Conflict of interest: None.

1 De Wals P, Hertoghe L, Borlee-Grimee I, De Maeyer-Cleempoel S, Reginster-Haneuse G, Dachy A, et al. Meningococcal disease in Belgium. Secondary attack rate among household, day-care nursery and pre-elementary school contacts. J Infect 1981;3(suppl 1):53-61S.

2 Meningococcal Disease Surveillance Group. Meningococcal disease. Secondary attack rate and chemoprophylaxis in the United States, 1974. JAMA 1976;235:261-5.

3 Olcén P, Kjellander J, Danielsson D, Lindquist BL. Epidemiology of Neisseria meningitidis: prevalence and symptoms from the upper respiratory tract in family members to patients with meningococcal disease. Scand J Infect Dis 1981;13:105-9.

4 Munford RS, Taunay AE, de Morais JS, Fraser DW, Feldman RA. Spread of meningococcal infection within household. Lancet 1974;i:1275-8.

5 Kristiansen BE, Tveten Y, Ask E, Rerten T, Knapskog A-B,Steen-Johnsen $\mathrm{J}$, et al. Preventing secondary cases of meningococcal disease by identifying and eradicating disease-causing strains in close contacts of patients. Scand J Infect Dis 1992;24:165-73.

6 Cartwright KAV, Stuart JM, Robinson PM. Meningococcal carriage in close contacts of cases. Epidemiol Infect 1991;106:133-41.

7 Cooke RPD, Riordan T, Jones DM, Painter MJ. Secondary cases of meningococcal infection among close family and household contacts in England and Wales, 1984-7. BMJ 1989;298:555-8.

8 Kristiansen BE, Fermér C, Jenkins A, Ask E, Swedberg G, Sköld O. PCR amplicon restriction endonuclease analysis of the chromosomal dhps gene of Neisseria meningitidis: a method for studying the spread of the disease-causing strain in contacts of patients with meningococcal disease. disease-causing strain in contacts of

9 Woods CR, Koeuth T, Estabrook MM, Lupski JR. Rapid determination of outbreak-related strains of Neisseria meningitidis by repetitive elementbased polymerase chain reaction genotyping. J Infect Dis 1996;174:760-7.

10 Caugant DA, Frøholm LO, Høiby EA, Blystad H, Lystad A. [abstract]. Improved surveillance of meningococcal disease in Norway by continual connection of the epidemiological and bacteriological data. Annual conference of the Institute of Public Health, Oslo, Norway, 1996. Oslo: Institute of Public Health.

11 Public Health Laboratory Service. Control of meningococcal disease: guidance for consultants in communicable disease control. Communicable disease report. PHLS Communicable Disease Surveillance Centre: London, 8 Dec, 1995.

12 Recommendation of the immunization practices advisory committee meningococcal vaccines. Centre for Disease Control. www.cdc.gov/ (1 April, 1996). 
13 Benenson AS. Control of communicable diseases manual, 16th ed. Washington DC: American Public Health Association, 1995.

14 Emerging and other communicable diseases. Meningococcal meningitis fact sheet (No 105). Geneva: World Health Organisation. www.who.ch programmes/emc/csmfacts.htm (Aug 3, 1996).

15 Pearson N, Gunnell DJ, Dunn C, Beswick T, Hill A, Ley B. Antibiotic prophylaxis for bacterial meningitis: overuse and uncertain efficacy. Public Health Med 1995;17:455-8.

16 Bøvre K, Gedde-Dahl TW. Epidemiological patterns of meningococcal disease in Norway 1975-1979. Natl Inst Public Health Ann, Oslo 1980;3:9 22.

17 Høiby EA, Moe PJ, Lystad A, Frøholm LO. Phenoxymethylpenicillin treatment of household contacts of meningococcal disease patients. Antonie van Leeuwenhoek J Microbiol 1986:52:255-7.

18 Kristiansen BE, Lind KW, Mevold K, Sørensen B, Frøholm LO, Bryn K, et al. Meningococcal carriage: studies of bacterial phenotypic and genomic characteristics and of human antibody levels. J Clin Microbiol 1988;26:1988-92.
19 Andersen BM. Mortality in meningococcal infections. Scand J Infect Dis 1978;10:277-82.

20 Reller LB, McGregor RR, Beaty HN. Bactericidal antibody after colonization with Neisseria meningitidis. J Infect Dis 1973;127:56-62.

21 Caugant DA, Høiby EA, Magnus P, Scheel O, Hoel T, Bjune G, et al. Asymptomatic carriage of Neisseria meningitidis in a randomly sampled population. J Clin Microbiol 1994:32:323-30.

22 Achtman M. Global epidemiology. In: Cartwright K, ed. Meningococcal dis ease. Chichester: John Wiley, 1995:159-76.

23 Cartwright K, Kroll S. Optimising the investigation of meningococcal disease. BMJ 1997;315:757-8.

24 Cartwright K. Meningococcal carriage and disease. In: Cartwright K, ed. Meningococcal disease Chichester: John Wiley, 1995:115-46.

25 Olcen P, Kjellander J, Danielsson D, Lindquist BL. Culture diagnosis of meningococcal carriers: yield from different sites and influence of storage in transport medium. J Clin Pathol 1979;32:1222-5.

(Accepted 6July 1998)

\title{
Effect of preventive treatment for tuberculosis in adults infected with HIV: systematic review of randomised placebo controlled trials
}

\author{
David Wilkinson, S B Squire, Paul Garner
}

\begin{abstract}
Objective: To determine whether preventive treatment for tuberculosis in adults infected with HIV reduces the frequency of tuberculosis and overall mortality.

Design: Systematic review and data synthesis of randomised placebo controlled trials.

Main outcome measures: Active tuberculosis, mortality, and adverse drug reaction requiring cessation of the study regimen. Outcomes stratified by status of purified protein derivative skin test. Results: Four trials comprising 4055 adults from Haiti, Kenya, the United States, and Uganda were included. All compared isoniazid (6-12 months) with placebo, and one trial also compared multidrug treatment for 3 months with placebo. Mean follow up was 15-33 months. Overall, frequency of tuberculosis (relative risk $0.57,95 \%$ confidence interval 0.41 to 0.79 ) was reduced in those receiving preventive treatment compared with placebo: mortality was not significantly reduced $(0.93,0.83$ to 1.05$)$. In subjects positive for purified protein derivative receiving preventive treatment, the risk of tuberculosis was reduced substantially $(0.32,0.19$ to 0.51$)$ and the risk of death was reduced moderately $(0.73,0.57$ to 0.95$)$ compared with those taking placebo. In adults negative for purified protein derivative receiving preventive treatment, the risk of tuberculosis $(0.82$, 0.50 to 1.36$)$ and the risk of death (1.02, 0.89 to 1.17$)$ were not reduced significantly. Adverse drug reactions were more frequent, but not significantly so, in patients receiving drug compared with placebo (1.45, 0.98 to 2.14).
\end{abstract}

Conclusions: Preventive treatment given for 3-12 months protects against tuberculosis in adults infected with HIV, at least in the short to medium term.

Protection is greatest in subjects positive for purified protein derivative, in whom death is also less frequent. Long term benefits remain to be shown.

\section{Introduction}

Strategies to control tuberculosis comprise case treatment, preventive treatment, and vaccination with $\mathrm{BCG}$, with the expectation that improved socioeconomic conditions will lead to a decline in disease incidence. ${ }^{12}$ Preventive treatment aims to eradicate latent infection with Mycobacterium tuberculosis before active disease develops. Latent infection is shown by a positive reaction to intradermal injection with purified protein derivative (tuberculin skin test). Trials in people with tuberculosis infection but not infected with HIV have shown that isoniazid given for 6-12 months substantially reduces the incidence of active tuberculosis. ${ }^{3}$

Infection with HIV has changed the natural history of infection with $M$ tuberculosis. ${ }^{4}$ People who are infected with HIV and who have a positive tuberculin skin test have a $30 \%$ or more lifetime risk of developing active tuberculosis, ${ }^{5}$ and tuberculosis is the most common HIV related disease in developing countries. $^{14}$ Thus, preventive treatment may be an important intervention to reduce the burden of tuberculosis in people infected with HIV, and their contacts, but its efficacy cannot simply be extrapolated from studies in people not infected with HIV.

As several fairly small trials have been done, we conducted this systematic review to summarise the evidence available to date as to whether preventive treatment for tuberculosis is effective in reducing the incidence of active tuberculosis and of death.

\section{Subjects and methods}

\section{Criteria for selecting studies for review}

We included only randomised controlled trials that compared drug regimens aimed at preventing tuberculosis with placebo. Trials were considered irrespective of setting or target group, and we included all different drug regimens tested. Preventive treatment was defined as tuberculosis chemotherapy given to people who have
Centre for Epidemiological Research in Southern Africa, Medical Research Council, PO Box 187, Mtubatuba 3935, South Africa David Wilkinson, specialist scientist

Tropical Medicine Division, Liverpool School of Tropical

Medicine, Liverpool L3 5QA

S B Squire,

senior lecturer

International Health Division, Liverpool School of Tropical Medicine Paul Garner, senior lecturer

Correspondence to: Dr Wilkinson wilkinsd@mrc.ac.za

BMJ 1998;317:625-9 